\title{
Excreção urinária de cálcio, ácido úrico e citrato em crianças $e$ adolescentes sadios
}

\author{
Urinary excretion of calcium, uric acid and citrate in healthy children and adolescents
}

\author{
Maria Goretti Moreira Guimarães Penido ${ }^{1}$, José Silvério Santos Diniz ${ }^{2}$, Milena Maria Moreira Guimarães ${ }^{3}$, \\ Rodrigo Barbosa Cardoso ${ }^{4}$, Marcelo Ferraz de Oliveira Souto ${ }^{5}$, Mariana Guimarães Penido 5
}

\section{Resumo}

Objetivo: determinar valores de referência regionais para a excreção urinária de cálcio, ácido úrico e citrato e estabelecer correlação entre essas excreções em urina em $24 \mathrm{~h}$ e amostras únicas, para uso na prática clínica.

Material e métodos: 125 crianças e adolescentes saudáveis, selecionados aleatoriamente, foram submetidos ao protocolo: exame clínico, bioquímica de sangue, hemograma, paratormônio, urina em $24 \mathrm{~h}$, urina em amostra única colhida com jejum, e exame parasitológico de fezes.

Resultados: o valor máximo para a excreção de cálcio em urina em $24 \mathrm{~h}$ foi $3,75 \mathrm{mg} / \mathrm{kg}$, em mg/dl do ritmo de filtração glomerular RFG foi 0,10 , e, para a relação cálcio/creatinina(mg/dl) na urina, em amostra única em jejum, foi de 0,25 . Observou-se correlação positiva entre a excreção de cálcio em urina em $24 \mathrm{~h}$, e urina em amostra única em jejum (mg/dl e mg/dl do RFG). Os valores máximos para a excreção de ácido úrico em urina em $24 \mathrm{~h}$ foram 600,450 e $320 \mathrm{mg}$ e 13,15 e $18 \mathrm{mg} / \mathrm{kg}$ para adolescentes, escolares e pré-escolares, respectivamente; em $\mathrm{mg} / \mathrm{dl}$ do RFG em amostra única de urina foi 0,47 . Observou-se correlação positiva para a excreção de ácido úrico em urina em $24 \mathrm{~h}$ e urina em amostra única em jejum. Os valores médios para a excreção de citrato em urina em $24 \mathrm{~h}$ foram 1,6, 1,1 e $0,5 \mathrm{mmol}$ para adolescentes, escolares e pré-escolares, respectivamente; para a relação citrato/creatinina em urina, em amostra única, com jejum, foi 0,3 .

Conclusões: as excreções de cálcio e ácido úrico em urina em 24h se correlacionaram com aquelas das amostras urinárias simplificadas, permitindo o uso destas para diagnósticos metabólicos, estudos populacionais e controle de pacientes hipercalciúricos e hiperuricosúricos sem controle de esfíncter vesical; o quociente citrato/creatinina em urina, em amostra única, pode ser utilizado para controle de pacientes com hipocitratúria.

J Pediatr (Rio J) 2002; 78 (2): 153-60: cálcio, ácido úrico, citrato e excreção urinária.

\begin{abstract}
Objective: to obtain regional reference values for calcium, uric acid and citrate urinary excretion and establish a correlation between those excretions in 24-hour urine sample and single urine sample for their use in clinical practice.
\end{abstract}

Methods: a hundred and twenty-five healthy children and adolescents were randomly chosen and submitted to the following protocol: clinical examination, biochemical analysis of blood, blood cell count, parathormone, 24-hour urine, fasting urine sample and stool test.

Results: the maximum value of calcium excretion in 24-hour urine was $3.75 \mathrm{mg} / \mathrm{kg}$; in $\mathrm{mg} / \mathrm{dl}$ of the glomerular filtration rate, it was 0.10 ; and for the calcium/creatinine $(\mathrm{mg} / \mathrm{dl})$ ratio in the fasting urine sample was 0.25 . Positive correlation was observed between calcium excretion in the 24-hour urine and the fasting sample $(\mathrm{mg} / \mathrm{dl}$ and $\mathrm{mg} / \mathrm{dl}$ of glomerular filtration rate). The maximum values of uric acid excretion in 24-hour urine were 600, 450, and 320mg and 13, 15 and $18 \mathrm{mg} / \mathrm{kg}$ for adolescents, school and preschool children, respectively; in $\mathrm{mg} / \mathrm{dl}$ of glomerular filtration rate, in the fasting urine sample, it was 0.47. Positive correlation was observed for the uric acid excretion in 24-hour urine and fasting urine samples. The mean values for the citrate excretion in 24-hour urine were 1.6, 1.1 and $0.5 \mathrm{mmol}$ for adolescents, school and preschool children, respectively; for citrate/creatinine ratio, in the fasting urine sample the mean value was 0.3 .

Conclusions: the calcium and uric acid excretion in 24-hour urine showed correlation with those in the fasting urine sample, which allows their use for metabolic diagnosis, population studies and follow-up of patients with hypercalciuria and hyperuricosuria without voiding control; the citrate/creatinine ratio in the fasting urine sample can be used for controlling patients with hypocitraturia.

J Pediatr (Rio J) 2002; 78(2): 153-60: calcium, uric acid, citrate and urinary excretion.

1. Doutora em Medicina: Pediatria - Faculdade de Medicina, Universidade Federal de Minas Gerais (UFMG).

2. Professor Adjunto - Faculdade de Medicina, UFMG.

3. Endocrinologista - Preceptora de Endocrinologia do IPSEMG.

4. Cardiologista com habilitação em Ergometria e Reabilitação Cardíaca.

5. Acadêmico da Faculdade de Medicina - UFMG.

Apoio: CAPES E CNPq.

Artigo submetido em 16.08.01, aceito em 12.12.01. 


\section{Introdução}

A hipercalciúria, a hiperuricosúria e a hipocitratúria idiopáticas são hoje reconhecidas como importantes fatores na patogênese da doença calculosa renal em adultos ${ }^{1,2}$ e crianças ${ }^{3-5}$.

Habitualmente, o diagnóstico destas hiper e hipoexcreções é feito em amostras urinárias colhidas durante 24 horas. A coleta desse tipo de amostra apresenta grandes dificuldades práticas, especialmente em pacientes pediátricos, podendo incorrer em erros e representar incômodo para a criança e ônus para a família. Os inconvenientes desse tipo de coleta levaram os pesquisadores a utilizarem os quocientes cálcio/creatinina ${ }^{6-8}$, ácido úrico/creatinina ${ }^{6,7,9}$ e citrato/creatinina ${ }^{10} \mathrm{em}$ amostras urinárias simplificadas ou avaliar a excreção desses elementos nessas mesmas amostras, corrigida pelo clearance de creatinina ou em $\mathrm{mg} / \mathrm{dl}$ do ritmo de filtração glomerular - $\mathrm{RFG}^{6,11,12}$.

O objetivo do estudo foi obter valores de referência para a excreção urinária de cálcio, ácido úrico e citrato relacionada à excreção de creatinina em amostras urinárias simplificadas, em crianças e adolescentes sadios, em região do sudeste do Brasil.

\section{Métodos}

Durante o período de coleta de dados foram avaliados 162 indivíduos. Destes, 4 foram excluídos do estudo porque tiveram diagnóstico de hiperexcreção urinária de cálcio, 3 porque tinham piúria no exame de urina, 8 porque não completaram todos os exames, e 22 porque não havia credibilidade na coleta do material. Assim, estudou-se uma amostra de 125 indivíduos de 2 a 18 anos, selecionados aleatoriamente, no Centro Social Urbano "Providência", do Distrito Sanitário Norte da Prefeitura Municipal de Belo Horizonte. Essa região engloba 48 bairros e comporta uma população de 162.575 habitantes, sendo 83.418 mulheres e 79.157 homens. Esses participantes foram selecionados dentre aqueles que buscavam assistência médico-odontológica, assistência social e assistência escolar, dentre outras, no referido Centro Social. Uma senha era distribuída a cerca de trinta indivíduos adultos que estavam acompanhados de crianças de 2 a 17 anos. Eram sorteados dez números, e as pessoas correspondentes eram convocadas para uma reunião na qual se explicava o motivo do sorteio. Após esclarecimentos detalhados, prestados aos familiares, sobre como se processaria o estudo, os concordantes eram agendados em grupos de três, e cada um destes três grupos participantes deveria comparecer ao Centro Social, às segundas e quartas-feiras, de acordo com agendamento prévio. Os responsáveis assinavam um termo de consentimento da entrada de suas crianças no estudo, declarando-se cientes de todos os procedimentos a serem realizados. No dia do sorteio, os indivíduos selecionados que concordaram em participar do estudo recebiam um impresso para responder a um inquérito alimentar. Esse inquérito, preparado por uma nutricionista, era feito no domicílio durante os três dias que antecediam a coleta do material. Posteriormente, era avaliado e analisado também pela mesma nutricionista. Quando na agenda já não havia paciente para a coleta de material, novos sorteios eram realizados sem dia ou qualquer data previstos antecipadamente. O número de desistências foi pequeno dentre aqueles sorteados e dentre aqueles já agendados para exame clínico e coleta de material. O percentual de desistência foi menor do que $10 \%$. Os familiares estavam cientes de que a continuidade da assistência médica estava garantida na eventualidade dessa necessidade.

A população estudada foi então composta por 125 indivíduos de 2 a 18 anos, representando três grupos etários, conforme Marcondes e colaboradores, $1991^{13}$. A amostra era constituída de 35\% de pré-escolares, 39\% de escolares e $26 \%$ de adolescentes. Todos foram mantidos em sua dieta, ingestão hídrica e atividades físicas habituais.

Para inclusão no estudo, os participantes, após exame clínico criterioso, deveriam ter pressão arterial normal, estar posicionados dentro das curvas de percentil 5 e 97 para peso e estatura para o sexo ${ }^{14}$, não utilizar preparados de cálcio, vitamina $\mathrm{D}$ ou drogas que alteram a excreção de ácido úrico no momento do estudo ou nos últimos dois meses, ter controle esfincteriano vesical diurno e noturno e não apresentar sintomatologia de endocrinopatias, nefropatias, patologias outras das vias urinárias ou patologias metabólicas.

De todos os indivíduos, foram coletados um espécime de sangue para hemograma e dosagem de: uréia, creatinina, cálcio, fósforo, fosfatase alcalina, ácido úrico, magnésio, cloro, sódio, potássio e paratormônio; 3 espécimes urinários: urina em 24 horas e urina em amostra única de $2^{\mathrm{a}}$ micção matinal com e sem jejum. Para a coleta de urina em 24 horas, os participantes eram mantidos sob vigilância de 07:00 às 19:00h e colhia-se num recipiente de vidro toda a urina do período diurno. No final do dia, cada participante recebia outro recipiente semelhante e, no domicílio, colhia a urina do período noturno. Na manhã seguinte, retornava trazendo o recipiente com a urina do período noturno, que era adicionada àquela do período diurno e homogeneizada. Colhia-se, então, a urina em amostra única matinal de $2^{\mathrm{a}}$ micção, sem jejum, em outro recipiente de vidro. A urina em amostra única matinal de $2^{\mathrm{a}}$ micção, com jejum, era colhida no dia seguinte, juntamente com o sangue.

Para conservação das amostras para dosagem de cálcio e citrato, utilizou-se ácido clorídrico P.A. a 50\% (v/v) por litro ${ }^{15,16} \mathrm{e}$, para dosagem de ácido úrico, utilizou-se bicarbonato de sódio P.A. $5 \mathrm{~g}$ por litro ${ }^{16}$.

Determinou-se a excreção urinária de cálcio em $\mathrm{mg} / \mathrm{kg} /$ $24 \mathrm{~h}$, de ácido úrico em $\mathrm{mg} / 24 \mathrm{~h}$ e $\mathrm{mg} / \mathrm{kg} / 24 \mathrm{~h}$ e citrato em $\mathrm{mmol} / 24 \mathrm{~h}$ e $\mathrm{mmol} / \mathrm{kg} / 24 \mathrm{~h}$. Nas amostras simplificadas (amostras únicas em $2^{\mathrm{a}}$ micção matinal com e sem jejum), determinou-se os quocientes: cálcio(mg/dl)/creatinina $(\mathrm{mg} / \mathrm{dl})$ e citrato( $\mathrm{mmol} / \mathrm{l}) /$ creatinina(mmol/l). Além disso, determinou-se em todas as amostras a excreção desses mesmos elementos em mg/dl RFG, segundo Simkin e 
colaboradores (1979) ${ }^{11}$. Segundo esses autores, é possível avaliar o clearance urinário de qualquer elemento em todos os tipos de amostras urinárias. O volume da amostra urinária nãoé um fator limitante, desde que se conheça os valores da creatinina e do elemento em estudo, na amostra urinária, e os valores da creatinina sérica. O importante é que a coleta de urina e sangue sejam feitas ao mesmo tempo. Assim sendo, a determinação da excreção dos referidos elementos em mg/dl RFG é de extrema importância, evitando os erros da coleta de urina em 24 horas, e possibilitando a avaliação da excreção desses mesmos elementos em crianças que ainda não apresentam controle esfincteriano diurno e/ou noturno.

O cálcio foi dosado por absorção atômica (aparelho Perkin-Elmer, 5000), o ácido úrico pelo método enzimático da urease (aparelho Cobas-Mira, Roche), a creatinina pelo método do picrato cinético (aparelho Cobas-Mira, Roche), e o citrato pelo método enzimático da citrato-liase ${ }^{10}$.

Um total de $84(67,2 \%)$ participantes do estudo responderam a um inquérito alimentar realizado durante três dias, no domicílio, para a análise dos valores médios diários da ingestão de proteínas, cálcio, sódio e calorias. Esses inquéritos foram analisados pelo Departamento de Nutrição e Dietética do Hospital das Clínicas da UFMG.
No estudo estatístico, determinou-se média, desviopadrão, $\mathrm{P}_{5}$ (percentil ${ }_{5}$ ) e $\mathrm{P}_{95}$ (percentil ${ }_{95}$ ), coeficiente de variação, análise de correlação de Pearson (r) e teste $t$ de Student.

Todos os procedimentos do estudo foram aprovados previamente pela Comissão de Ética em Pesquisa da Faculdade de Medicina da Universidade Federal de Minas Gerais.

\section{Resultados}

A análise bioquímica do sangue, assim como a análise bioquímica urinária, o volume de urina em $24 \mathrm{~h}$, as sedimentoscopias, densidades e $\mathrm{pH}$ urinários não revelaram alterações.

Comparou-se os resultados obtidos do inquérito alimentar com aqueles recomendados pelo RDA ${ }^{14}$, e observou-se que as quantidades médias de ingestão para o cálcio (mg/dia) e calorias (kcal/dia) estavam abaixo daquelas recomendadas em todas as faixas etárias. Entretanto, as quantidades médias obtidas para a ingestão de sódio $(\mathrm{mg} /$ dia) e proteínas ( $\mathrm{g} / \mathrm{dia}$ ) superaram os padrões estabelecidos e recomendados para todas as faixas etárias (Tabela 1).

Tabela 1 - Valores médios diários para a ingestão de calorias, cálcio, sódio e proteínas na população estudada e os valores recomendados pelo RDA, $\mathrm{n}=87$

\begin{tabular}{|c|c|c|c|c|c|}
\hline Variável & Faixa etária (anos) & Mínimo & Máximo & Média $\pm D . P . *$ & $\mathbf{R D A}^{\dagger}$ \\
\hline \multirow{7}{*}{ Calorias (Kcal/24h) } & 1 a 3 & 986,6 & $1.540,2$ & $1.194,9 \pm 303,8$ & 1.300 \\
\hline & 4 a 6 & 817,9 & $1.414,1$ & $1.105,6 \pm 228,6$ & 1.800 \\
\hline & 7 a 10 & $1.013,5$ & $2.255,7$ & $1.544,2 \pm 439,3$ & 2.000 \\
\hline & 11 a 14 - masc. & $1.118,7$ & $1.364,3$ & $1.255,5 \pm 125,1$ & 2.500 \\
\hline & 11 a 14 - fem. & $1.170,3$ & $1.534,8$ & $1.318,7 \pm 156,9$ & 2.200 \\
\hline & 15 a 18 - masc & $2.708,2$ & $2.708,2$ & $2.708,2 \pm-$ & 3.000 \\
\hline & 1 a 3 & 269,3 & 618,1 & $407,7 \pm 185,2$ & 800 \\
\hline \multirow{5}{*}{ Cálcio (mg/24h) } & 4 a 6 & 186,4 & 678,0 & $426,8 \pm 151,1$ & 800 \\
\hline & 7 a 10 & 201,3 & 640,9 & $479,6 \pm 127,3$ & 800 \\
\hline & 11 a 14 - masc. & 272,9 & 303,1 & $288,5 \pm 15,1$ & 1.200 \\
\hline & 11 a 14 - fem. & 155,4 & 454,9 & $305,6 \pm 132,1$ & 1.200 \\
\hline & 15 a 18 - masc & 917,5 & 917,5 & $917,5 \pm-$ & 1.200 \\
\hline \multirow{6}{*}{ Sódio (mg/24h) } & 1 a 3 & 826,3 & $2.713,4$ & $1.680,1 \pm 956,2$ & 225 \\
\hline & 4 a 6 & 756,4 & $2.543,3$ & $1.460,3 \pm 578,3$ & 300 \\
\hline & 7 a 10 & 798,7 & $4.816,3$ & $2.178,0 \pm 1.012,5$ & 400 \\
\hline & 11 a 14 - masc. & $1.154,7$ & $1.947,4$ & $1.769,1 \pm 198,8$ & 500 \\
\hline & 11 a 14 - fem. & $1.876,0$ & $2.770,7$ & $2.201,4 \pm 392,4$ & 500 \\
\hline & 15 a 18 - masc & $4.104,1$ & $4.104,1$ & $4.104,1 \pm-$ & 500 \\
\hline \multirow{6}{*}{ Proteína (g/24h) } & 1 a 3 & 31,6 & 60,5 & $45,7 \pm 14,5$ & 16 \\
\hline & 4 a 6 & 35,7 & 56,4 & $43,2 \pm 7,7$ & 24 \\
\hline & 7 a 10 & 22,7 & 88,2 & $57,7 \pm 19,6$ & 28 \\
\hline & 11 a 14 - masc. & 35,9 & 47,6 & $41,3 \pm 5,9$ & 45 \\
\hline & 11 a 14 - fem. & 34,6 & 67,1 & $46,3 \pm 14,8$ & 46 \\
\hline & 15 a 18 - masc & 114,3 & 114,3 & $114,3 \pm-$ & 59 \\
\hline
\end{tabular}

\footnotetext{
* Desvio-padrão † Recocommended dietary alllowances

Na faixa de 15 a 18 anos, apenas um participante respondeu ao inquérito
} 
Estudou-se também a correlação entre as quantidades médias diárias ingeridas e excretadas na urina em $24 \mathrm{~h}$, de cálcio, proteína e sódio. Obteve-se correlação positiva entre a excreção de sódio e a de cálcio $(r=0,74 ; \mathrm{p}<0,01)$. Nenhuma correlação foi encontrada para a excreção de proteína $(\mathrm{r}=0,26 ; \mathrm{p}=0,16)$ e excreção de cálcio $(\mathrm{r}=0,23$; $\mathrm{p}=0,21)$.

O valor máximo $\left(\mathrm{P}_{95}\right)$ para a excreção de cálcio em urina em 24 horas foi $3,75 \mathrm{mg} / \mathrm{kg}$. Para o quociente cálcio/creatinina em amostra única, com jejum, o valor máximo foi 0,25 , e sem jejum foi 0,32. O estudo da excreção de cálcio em mg/ dl do RFG $^{11,18}$ teve o valor máximo de 0,10 em amostra de 24 horas, 0,10 em amostra única com jejum, e 0,12 naquela sem jejum (Tabela 2). Os valores mínimos e máximos da excreção de cálcio para as diferentes amostras urinárias estão apresentados na Tabela 2 .

Não houve diferenças estatísticas para excreção de cálcio em relação às faixas etárias e sexos. A prevalência da hipercalciúria idiopática foi $3,2 \%$,

Tabela 2 - Excreção de cálcio em urina em $24 \mathrm{~h}$ e urina em amostra única, $\mathrm{n}=125$

\begin{tabular}{|c|c|c|c|c|}
\hline Amostras & Mínimo & Máximo & Média $\pm D P *$ & $\mathbf{P}_{\mathbf{9 5}}$ \\
\hline $\mathrm{mg} / \mathrm{kg} / 24 \mathrm{~h}$ & 0,02 & 6,80 & $1,44 \pm 1,17$ & 3,75 \\
\hline $\begin{array}{l}\text { amostra única } \\
\text { com jejum }\end{array}$ & 0,004 & 0,92 & $0,10 \pm 0,10$ & 0,25 \\
\hline $\begin{array}{l}\text { amostra única } \\
\text { sem jejum }\end{array}$ & 0,002 & 0,55 & $0,10 \pm 0,10$ & 0,32 \\
\hline urina $24 \mathrm{~h}(\mathrm{mg} / \mathrm{dl} \mathrm{RFG})$ & 0,001 & 0,48 & $0,04 \pm 0,04$ & 0,10 \\
\hline $\begin{array}{l}\text { amostra única } \\
\text { com jejum (mg/dl RFG) }\end{array}$ & 0,0004 & 0,34 & $0,03 \pm 0,03$ & 0,10 \\
\hline $\begin{array}{l}\text { amostra única } \\
\text { sem jejum (mg/dl RFG) }\end{array}$ & 0,001 & 0,22 & $0,04 \pm 0,04$ & 0,12 \\
\hline
\end{tabular}

Observou-se correlação positiva entre a excreção de cálcio total (mg) na amostra de 24 horas e a relação da excreção cálcio/creatinina $(\mathrm{mg} / \mathrm{dl})$ nas amostras únicas, com jejum e sem jejum. Houve também correlação positiva entre a excreção de cálcio em mg/dl do RFG em amostra de 24 horas e nas urinas de amostra única, com jejum e sem jejum (Tabela 3).

Tabela 3 - Correlação entre excreção de cálcio em urina 24h e amostra única simplificada

\begin{tabular}{lccc}
\hline Amostra urina 24h & $\begin{array}{c}\text { Amostra única urina } \\
\text { com jejum }\end{array}$ & $\mathbf{r}^{*}$ & $\mathbf{p}^{\dagger}$ \\
\hline Cálcio mg/24 h & cálcio/creatinina (mg/dl) & 0,22 & $<0,02$ \\
Cálcio mg/dl RFG & cálcio (mg/100ml RFG) & 0,24 & 0,02 \\
\hline
\end{tabular}

* coeficiente de correlação † probabilidade de significância

O estudo da excreção de ácido úrico em urina em 24 horas (mg e mg/kg) e do quociente ácido úrico/creatinina nas amostras únicas, com e sem jejum, mostraram valores diversos nas diferentes faixas etárias, e não houve correlação entre os mesmos. Os valores máximos $\left(\mathrm{P}_{95}\right)$ para a excreção de ácido úrico em urina em $24 \mathrm{~h}$ foram $600,450 \mathrm{e}$ $320 \mathrm{mg}$ e 13,15 e $18 \mathrm{mg} / \mathrm{kg}$ para adolescentes, escolares e pré-escolares, respectivamente (Tabela 4). Na avaliação da excreção de ácido úrico em $\mathrm{mg} / \mathrm{dl}$ do RFG, obteve-se o valor máximo $\left(\mathrm{P}_{95}\right)$ de 0,45 na amostra de $24 \mathrm{~h}, 0,47 \mathrm{na}$ amostra única com jejum e 0,43 naquela sem jejum (Tabela 4). Os valores mínimos e máximos da excreção de ácido úrico para as diferentes amostras urinárias estão apresentados na Tabela 4. Houve correlação positiva entre os valores de ácido úrico em mg/dl do RFG encontrados na amostra de $24 \mathrm{~h}$ e nas amostras únicas, com jejum e sem jejum, em

Tabela 4 - Excreção urinária de ácido úrico em mg, m/kg e mg/dl RFG, n = 125

\begin{tabular}{llcccc}
\hline Amostras & & Mínimo & Máximo & Média \pm DP* & $\mathbf{P}_{\mathbf{9 5}}{ }^{\dagger}$ \\
\hline \multirow{2}{*}{$24 \mathrm{~h}(\mathrm{mg})$} & Adolescente & 68,0 & 842,8 & $380,0 \pm 145,0$ & 600,0 \\
& Escolar & 15,0 & 526,4 & $270,0 \pm 110,0$ & 450,0 \\
& Pré-escolar & 25,2 & 362,5 & $175,0 \pm 78,0$ & 320,0 \\
& Adolescente & 1,5 & 17,0 & $7,0 \pm 3,0$ & 13,0 \\
$24 \mathrm{~h}(\mathrm{mg} / \mathrm{kg})$ & Escolar & 1,0 & 19,3 & $9,0 \pm 4,0$ & 15,0 \\
& Pré-escolar & 2,0 & 31,3 & $10,5 \pm 5,0$ & 18,0 \\
& & & & & \\
$24 \mathrm{~h}(\mathrm{mg} / \mathrm{dl}$ RFG) & 0,001 & 3,6 & $0,25 \pm 0,36$ & 0,45 \\
Única com jejum (mg/dl RFG) & 0,04 & 0,8 & $0,26 \pm 0,13$ & 0,47 \\
Única sem jejum (mg/dl RFG) & 0,05 & 1,1 & $0,24 \pm 0,14$ & 0,43
\end{tabular}

\footnotetext{
* Desvio-padrão $\quad \dagger$ Percentil 95
} 
mg/dl do RFG. (Tabela 5). Não houve diferenças estatísticas para excreção de ácido úrico em mg/dl do RFG em relação às faixas etárias e sexos.

$\mathrm{O}$ valor mínimo $\left(\mathrm{P}_{5}\right)$ para a excreção urinária de citrato em $\mathrm{mmol} / 24 \mathrm{~h}$ foi $0,30,0,20 \mathrm{e} 0,02 \mathrm{mmol}$ para adolescentes, escolares e pré-escolares, respectivamente; e o valor médio foi $1,6 \pm 1,0,1,1 \pm 0,7$ e $0,5 \pm 0,5$ para adolescentes, escolares e pré-escolares, respectivamente (Tabela 6). O valor míni$\mathrm{mo}\left(\mathrm{P}_{5}\right)$ para a excreção urinária de citrato em $\mathrm{mmol} / \mathrm{kg} / 24 \mathrm{~h}$ foi $0,01,0,01$ e 0,002 para adolescentes, escolares e préescolares, respectivamente; e o valor médio foi $0,03 \pm 0,02$, $0,04 \pm 0,03$ e $0,03 \pm 0,02$ para adolescentes, escolares e préescolares, respectivamente (Tabela 6).

Não houve diferenças estatísticas para excreção de citrato em $\mathrm{mg} / \mathrm{dl}$ do $\mathrm{RFG}$ em relação às faixas etárias e sexos. O valor mínimo $\left(\mathrm{P}_{5}\right)$ para excreção de citrato em $\mathrm{mmol} / \mathrm{dl}$ do RFG foi 0,013 na amostra de $24 \mathrm{~h}$, e o valor médio para essa mesma excreção foi $0,19 \pm 0,17$ (Tabela 6). $\mathrm{O}$ valor mínimo $\left(\mathrm{P}_{5}\right)$ para excreção de citrato em $\mathrm{mmol} / \mathrm{dl}$ do RFG foi 0,015 na amostra única com jejum, e 0,022 na sem jejum. Os valores médios para esta mesma excreção foram $0,18 \pm 0,18$ na amostra única com jejum, e $0,17 \pm 0,17$ na sem jejum (Tabela 6). Para o quociente citrato/creatinina $(\mathrm{mmol} / \mathrm{l})$, o valor mínimo $\left(\mathrm{P}_{5}\right)$ foi 0,02 na amostra única com jejum, e 0,04 na sem jejum. Os valores médios para o mesmo quociente foram $0,3 \pm 0,3$ na amostra única com jejum e sem jejum. Não houve correlação entre a excreção de citrato na amostra de 24 horas e nas amostras urinárias únicas (Tabela 6). Os valores mínimos e máximos da excreção de citrato para as diferentes amostras urinárias estão apresentados na Tabela 6. Fatores como cor da pele,
Tabela 5 - Correlação entre excreção de ácido úrico em urina $24 \mathrm{~h}$ e amostra única simplificada

\begin{tabular}{llll}
\hline & Amostras urinárias & $\mathbf{r}^{*}$ & $\mathbf{p}^{\dagger}$ \\
\hline Excreção ácido úrico 24h & Única com jejum & 0,48 & $<\mathbf{0 , 0 1}$ \\
& Única sem jejum & 0,67 & $<\mathbf{0 , 0 1}$ \\
\hline${ }^{*}$ coeficiente de correlação & † probabilidade de significância &
\end{tabular}

sexo e história familial positiva para litíase renal não alteraram a excreção de cálcio, ácido úrico e citrato nas diferentes amostras urinárias estudadas.

\section{Discussão}

Hiper e hipoexcreções como hipercalciúria, hiperuricosúria e hipocitratúria estão freqüentemente associadas à doença litiásica renal ${ }^{1-3,5,19,20,22}$. Torna-se fundamental o reconhecimento dessas alterações metabólicas para que se possa atuar de maneira objetiva na prevenção da formação lítica. Essa prevenção poderia acontecer a partir da avaliação, controle e seguimento dos pacientes potencialmente formadores de cálculos ${ }^{23}$, assim como a partir do conhecimento que ora se tem dessas alterações metabólicas e dos valores considerados normais para a excreção urinária desses elementos potencialmente litogênicos.

Tabela 6 - Excreção urinária de citrato em mmol $/ 24 \mathrm{~h}, \mathrm{mmol} / \mathrm{kg} / 24 \mathrm{~h}, \mathrm{mmo} / \mathrm{dl}$ RFG e quociente citrato/ creatinina $(\mathrm{mmol} / \mathrm{l}), \mathrm{n}=125$

\begin{tabular}{|c|c|c|c|c|c|}
\hline \multicolumn{2}{|l|}{ Amostras } & \multirow{2}{*}{$\begin{array}{c}\text { Mínimo } \\
0,07\end{array}$} & \multirow{2}{*}{$\begin{array}{c}\text { Máximo } \\
4,17\end{array}$} & \multirow{2}{*}{$\begin{array}{c}\text { Média } \pm \mathbf{D P} * \\
1,6 \pm 1,0\end{array}$} & \multirow{2}{*}{$\frac{\mathbf{P}_{\mathbf{5}}^{\dagger}}{0,30}$} \\
\hline & Adolescente & & & & \\
\hline $\mathrm{mmol} / 24 \mathrm{~h}$ & Escolar & 0,03 & 2,77 & $1,1 \pm 0,7$ & 0,20 \\
\hline & Pré-escolar & 0,02 & 2,27 & $0,5 \pm 0,5$ & 0,02 \\
\hline \multirow{3}{*}{$\mathrm{mmol} / \mathrm{kg} / 24 \mathrm{~h}$} & Adolescente & 0,002 & 0,08 & $0,03 \pm 0,02$ & 0,01 \\
\hline & Escolar & 0,001 & 0,10 & $0,04 \pm 0,03$ & 0,01 \\
\hline & Pré-escolar & 0,001 & 0,10 & $0,03 \pm 0,02$ & 0,002 \\
\hline \multirow{3}{*}{\multicolumn{2}{|c|}{$\begin{array}{l}\text { 24h (mg/dl RFG) } \\
\text { Única com jejum (mg/dl RFG) } \\
\text { Única sem jejum (mg/dl RFG) }\end{array}$}} & 0,004 & 1,36 & $0,19 \pm 0,17$ & 0,013 \\
\hline & & 0,006 & 1,40 & $0,18 \pm 0,18$ & 0,015 \\
\hline & & 0,006 & 3,86 & $0,17 \pm 0,17$ & 0,022 \\
\hline \multirow{2}{*}{\multicolumn{2}{|c|}{$\begin{array}{l}\text { Citrato/creatinina com jejum }(\mathrm{mmol} / \mathrm{l}) \\
\text { Citrato/creatinina sem jejum }(\mathrm{mmol} / \mathrm{l})\end{array}$}} & 0,01 & 2,26 & $0,3 \pm 0,3$ & 0,02 \\
\hline & & 0,01 & 2,55 & $0,3 \pm 0,3$ & 0,04 \\
\hline
\end{tabular}


O resultado obtido para a excreção de cálcio em urina, em 24 horas, em mg/kg, comprovou o que já é consenso na comunidade científica mundial ${ }^{6-8,24,25}$. A coleta de urina em amostra única, com jejum noturno, foi utilizada por diversos autores ${ }^{6,24-26}$. A partir dela, tem-se encontrado valores limites para a excreção de cálcio em várias regiões do mundo. Acredita-se que o valor obtido para o quociente cálcio/creatinina seja representativo daquele valor encontrado em urina, em $24 \mathrm{~h}^{6,17}$. $\mathrm{O}$ valor do $\mathrm{P}_{95}$ encontrado no estudo para a relação cálcio/creatinina em urina, em amostra única, matinal, colhida com jejum noturno, foi 0,25 , valor próximo ao encontrado por alguns autores ${ }^{6,24}$. Outros pesquisadores encontraram valores um pouco menores, como $0,21^{26}$ e $0,22^{25}$. Possivelmente, a discreta elevação nos índices encontrados para a relação cálcio/creatinina em urina, em amostra única, matinal, colhida com jejum noturno, estaria relacionada com hábitos nutricionais ou fatores constitucionais. Segundo o inquérito alimentar realizado pelos participantes do estudo, ficou demonstrado que os valores para a ingestão protéica e sódica eram maiores, e os valores para a ingestão calórica e cálcica eram menores do que aqueles recomendados pelo $\mathrm{RDA}^{13}$ (Tabela 1). É sabido que a ingestão elevada de sódio induz hipercalciúria em pessoas sadias ${ }^{30}$. A relação entre natriúria e calciúria se interpreta com sendo secundária ao manejo de cálcio e sódio pelo túbulo proximal renal. Autores têm demonstrado correlação positiva entre natriúria e calciúria $25,26,30,32$. Essa correlação foi também encontrada no estudo ( $\mathrm{p}<0,01$; $r=0,74)$. Assim, provavelmente, o maior valor para o $\mathrm{P}_{95}$ para o quociente cálcio/creatinina encontrado para a urina em amostra única, matinal, colhida com jejum noturno, estaria ligada ao hábito do povo brasileiro de ingerir grande quantidade de sal na dieta.

De acordo com Peacock e colaboradore ${ }^{27}$ e Torres e colaboradores $^{28}$, o conteúdo de cálcio da dieta não parece aumentar de maneira apreciável a calciúria de pessoas sadias. Dauncey \& Widdowson, em $1972^{29}$, afirmaram que o conteúdo de cálcio na água habitualmente utilizada para beber também não influiria na calciúria de pessoas sadias. Stapleton e colaboradores ${ }^{26}$, em 1982, concluíram que a restrição dietética de produtos derivados do leite não tinham efeito significativo na relação cálcio/creatinina de urina em amostra única, colhida com jejum, de indivíduos sadios. À semelhança desses autores, encontrou-se no estudo esses mesmos resultados, indicando que uma maior ingestão de cálcio não corresponde a uma maior excreção urinária desse elemento $(\mathrm{r}=0,23 ; \mathrm{p}=0,23)$.

Em 1967, Nordin e colaboradores ${ }^{18}$ fizeram referência ao estudo da excreção de cálcio corrigida pelo clearance de creatinina ou em $\mathrm{mg} / \mathrm{dl}$ do RFG, demonstrando que os resultados obtidos expressavam realmente o ritmo de excreção de cálcio. Baseando-se nessa afirmativa, estudou-se a excreção de cálcio corrigida pelo clearance de creatinina em todas as amostras urinárias, e foi demonstrada correlação positiva entre os valores encontrados na amostra de urina em $24 \mathrm{~h}$ e aqueles das amostras únicas (Tabela 3). Em relação à amostra urinária única, matinal, sem jejum, apesar dos resultados para correlação encontrados serem positivos, é pouco utilizada rotineiramente e pouco mencionadas pelos autores ${ }^{33,34}$.

A prevalência da hipercalciúria assintomática encontrada no estudo foi 3,2\%, próxima daquelas encontradas por Moore e colaboradores ${ }^{31}$ e Kruse e colaboradores ${ }^{25}$. Entretanto, bem menores que aquelas encontradas por Perrone e colaboradores $^{21}$ e Hernandez-Marco e colaboradores ${ }^{6}$.

A avaliação da excreção de ácido úrico foi feita nas mesmas amostras urinárias descritas para a excreção de cálcio, entretanto os resultados foram variados, principalmente em relação à idade, peso corporal e estatura, e não se obteve correlação entre os valores obtidos nas amostras de urina em 24 horas e aqueles obtidos do quociente ácido úrico/creatinina $(\mathrm{mg} / \mathrm{dl})$. Essa heterogeneidade de resultados reflete a grande variabilidade da excreção urinária de ácido úrico, que estaria relacionada com hábitos alimentares, ou seja, relação direta entre elevada ingestão de proteína e aumento da excreção urinária de ácido úrico, como descrito por Zollner, em $1973^{35}$. No estudo, não foi encontrada esta relação direta entre ingestão elevada de proteínas e excreção urinária de ácido úrico aumentada. Assim sendo, continua polêmica, entre os pesquisadores, a melhor maneira de se expressar a excreção urinária de ácido úrico. Possivelmente, as mensurações mais acuradas seriam aquelas corrigidas pela superfície corpórea ${ }^{36,37}$ ou corrigidas por decilitro do clearance de creatinina ( $\mathrm{mg} / \mathrm{dl} \mathrm{RFG}$ ), como primeiramente proposto por Simkin e colaboradores ${ }^{11}$. No estudo, foi avaliada a excreção de ácido úrico em $\mathrm{mg} / \mathrm{dl}$ do RFG, e observou-se que fatores como idade, peso corpóreo e estatura não influenciaram o valor dessa excreção. Além do mais, a mensuração da excreção de ácido úrico poderia ser feita em amostras urinárias únicas, dispensando coletas de urina em 24 horas, que são muito trabalhosas. Correlação positiva foi obtida entre os valores para excreção de ácido úrico em $\mathrm{mg} / \mathrm{dl}$ do $\mathrm{RFG}$ em urina em $24 \mathrm{~h}$ e aqueles valores obtidos para as amostras urinárias únicas, matinais, colhidas com e sem jejum noturno (Tabela 5).

Infelizmente, o estudo da citratúria ainda não figura na rotina das avaliações metabólicas de pacientes litiásicos. Com base no significante papel que esse elemento desempenha na urina, estudou-se sua excreção em urina em $24 \mathrm{~h}$ e urinas de amostra única matinal, com e sem jejum noturno (Tabela 6). É interessante citar que os valores para excreção de citrato em $\mathrm{mmol} / \mathrm{kg} / 24 \mathrm{~h}$ não variaram com a idade $(\mathrm{p}=0,21)$, assim como os valores do quociente citrato/ creatinina em urinas de amostra única, matinal, com jejum $(\mathrm{p}=0,36)$ e sem jejum $(\mathrm{p}=0,22)$. Diferentemente, houve variações na excreção de citrato em mmol/24h $(\mathrm{p}<0,001)$ em relação à idade, peso e estatura, de acordo com o encontrado por Schwille e colaboradores ${ }^{38}$.

Pouco se sabe sobre o quociente citrato/creatinina de amostra urinária única, matinal. Alguns autores estudaram esse quociente em urina em $24 \mathrm{~h}$ de adultos ${ }^{39,40} \mathrm{e}$, em 1992, Trinchieri e colaboradores ${ }^{10}$ obtiveram valores diferentes 
para esse quociente em relação ao sexo e à idade. No estudo, não foram observadas essas variações. Interessante notar que quando se comparou os valores médios de excreção de citrato em urina em $24 \mathrm{~h} \mathrm{em} \mathrm{mmol} / 24 \mathrm{~h}$ e aqueles do quociente citrato/creatinina nas urinas de amostra única com e sem jejum (Tabela 5), não se observou correlação entre as mesmas, apesar de que os valores obtidos nessas amostras não apresentaram diferenças estatisticamente significativas $(\mathrm{p}<0,001)$. A interpretação desse fato ainda passa distante do entendimento sobre excreção de citrato.

Apesar de que nenhuma referência tenha sido encontrada na literatura sobre excreção de citrato em mg/dl do RFG, ela foi avaliada e observou-se que fatores como idade e sexo não alteravam os valores encontrados nas amostras urinárias. Diferentemente do encontrado para o cálcio e ácido úrico, nenhuma correlação foi obtida entre a excreção de citrato na urina em $24 \mathrm{~h}$ e as amostras urinárias únicas.

Após todos esses estudos, avaliações quantitativas e observações clínicas, entendeu-se que as excreções de cálcio, ácido úrico e citrato deveriam ser analisadas em populações de maiores proporções e de regiões geográficas diferentes. Apesar disso, pode-se concluir que:

1. a excreção do cálcio em urina em 24 horas correlacionou-se com a relação da excreção de cálcio/creatinina e com a excreção do cálcio em mg/dl do RFG (corrigida pelo clearance de creatinina) em amostras urinárias simplificadas, permitindo o uso destas em estudos populacionais, no diagnóstico e controle dos indivíduos sabidamente hipercalciúricos;

2. a excreção de ácido úrico na urina em 24 horas e nas amostras simplificadas foi variável, não se tendo obtido correlação entre os valores dessas amostras. Entretanto, houve correlação positiva entre os valores obtidos na urina em 24 horas e nas amostras únicas matinais, quando avaliadas em $\mathrm{mg} / \mathrm{dl}$ do RFG. Esses achados, que se correlacionaram, permitem o uso das amostras únicas matinais, em estudos populacionais, no diagnóstico e controle dos indivíduos sabidamente hiperuricosúricos;

3. conforme os estudos realizados, a excreção de citrato em urina em 24 horas não pode ser substituída pelos valores obtidos nas amostras únicas, já que não houve correlação entre os valores das mesmas. Entretanto, o quociente citrato/creatinina em urina em amostra única pode ser utilizado para controle de pacientes com hipocitratúria.

\section{Agradecimentos}

Agradecemos a todos os funcionários do Laboratório Central da FM UFMG, a Milton Batista Franco, pela dosagem de todo o material do estudo, à nutricionista Ermelinda Maria Leite Prado Lara e a todos os colegas do grupo de nefrologia pediátrica do Hospital das Clínicas da FM UFMG, em especial aos professores Maria Lúcia
Soares Ferreira Moreira, Eleonora Moreira Lima, Luiz Sérgio Bahia Cardoso e José Maria Penido Silva. A todas as crianças que participaram do estudo, o nosso carinho e respeito.

\section{Referências bibliográficas}

1. Pak CYC. Etiology and treatment of urolithiasis. Am J Kidney Dis 1999;18:624-37.

2. Coe FL, Parks JH, Asplin JR. The pathogenesis and treatment of kidney stones. N Engl J Med 1992;327:1141-51.

3. Stapleton FB, Mackay MP, Noe HN. Urolithiasis in children: the role of hypercalciuria. Pediatr Annals 1987;16:980-97.

4. Perrone HC, Sigulen D, Toporovski J, Schor N. Normatização da excreção urinária de cálcio e ácido úrico em crianças. J Bras Nefrol 1990;12:23-8.

5. Nicar MJ, Skurla C, Sakhaee K, Pak CYC. Low urinary citrate excretion in nephrolithiasis. Urology 1983;21:8-14.

6. Hernandez-Marco R, Nuñez-Gomes F, Martinez-Costa C, FonsMoreno J, Peris-Vidal A, Brines-Solanes J. Excreción urinaria de calcio, magnesio, ácido úrico y ácido oxálico en niños normales. An Esp Pediatr 1988;29[Suppl 2]:99-104.

7. De Santo NG, Di Iorio, Capasso G, Paduano C, Stamler R, Langman CB, et al. Population based data on urinary excretion of calcium, magnesium, oxalate, phosphate and uric acid in children from Cimitile (southern Italy). Pediatr Nephrol 1992;6:149-57.

8. Chen YK, Lee AJ, Chesney RW, Stapleton, FB, Roy S III. Urinary mineral excretion among Taiwanese children. Pediatr Nephrol 1994;8:36-40.

9. Kauffman JM, Green ML, Seegmiller JE. Urine uric acid to creatinine ratio - a screening test for inherited disorder of purine metabolism. J Pediatr 1968;73:583-92.

10. Trinchieri A, Mandressi A, Luongo P, Rovera F, Longo G. Urinary excretion of citrate, glycosamonoglycans, magnesium and zinc in relation to age and sex in normal subjects and in patients who form calcium stones. Scand J Urol Nephrol 1992;26:379-86.

11. Simkin PA, Hoover PL, Paxson CS, Wilson WF. Uric acid excretion: quantitative assessment for spot, midmorning serum urine samples. An Int Med 1979;91:44-7.

12. Stapleton FB, Nash D. A screening test for Hyperuricosuria. J Pediatr 1983;102:88-90.

13. Marcondes E, Machado DVM, Setian N. Crescimento e desenvolvimento. In: Marcondes E, ed. Pediatria Básica. $8^{\mathrm{a}}$ ed. São Paulo: Savier; 1991. p.1790.

14. National Center for Health Statistics. Growth curves for children birth - 18 years (Vital and health statistics. Series 11:165). Washington: National Center for Health Statistics; 1977.

15. Tiselius HG. Practical aspects of the handling of samples and evaluation of laboratory data. Scan J Urol Nephrol 1980;53:105-8.

16. Berg C, Larson L, Tiselius HG. The composition of four-hour urine samples from patients with calcium oxalate stone disease. Br J Urol 1987;60:301-6.

17. Soliman SA, Abdel-Hay MH, Sulaiman MI, Tayeb OS. Stability of creatinine, urea and uric acid stored under various conditions. Clin Chim Acta 1986;160:319-26.

18. Nordin BEC, Hodgkinson A, Peacock M. The measurement and meaning of urinary calcium. Clin Orthop 1967;52:293-322.

19. Andres A, Praga M, Bello I, Diaz-Rolón JÁ, Gutierrez-Millet V, Morales JM, et al. Hematuria due to hypercalciuria and hyperuricosuria in adults patients. Kidney Int 1989;36:96-9. 
20. Stapleton FB. Idiopathic hypercalciuria: association with isolated hematuria and risk for urolithiasis in children. The southwest pediatric nephrology study group. Kidney Int 1990;37: 807-11.

21. Perrone, HC, Ajzen H, Toporovski J, Schor N. Associação de hematúria e hipercalciúria na infância. Rev Ass Med Bras 1988;34:129-32.

22. Conte A, Roca P, Gianotti M, Grases F. On the relation between citrate and calcium in normal and stone-former subjects. Int Urol Nephrol 1989;21[Suppl 4]:369-73.

23. Santos MVA, Santos DR, Pinheiro ME, Perrone HC, Toporovski J, Schor N. Avaliação prospectiva do tratamento e acompanhamento ambulatorial de crianças com urolitíase. J Bras Pediatr 1987;63[Suppl 5]:247-50.

24. Ghazali S, Barratt TM. Urinary excretion of calcium and magnesium in children. Arch Dis Child 1947;49:97-101.

25. Kruse K, Kracht V, Kruse U. Reference values for urinary calcium excretion and screening for hypercalciuria in children and adolescents. Eur J Pediatr 1984;143:25-31.

26. Stapleton FB, Norman NH, Roy III S, Jerkins G. Hypercalciuria in children with urolithiasis. Am J Dis Child 1982;136:675-8.

27. Peacock M, Nordin BEC, Hodgkinson A. Importance of dietary calcium in definition of hypercalciuria. Br Med J 1967;3:469-71.

28. Torres RC, del Rio SS, Zuluaga GA, Espejo ME, Puebla CM. Influencia del calcio de la dieta en la incidencia de litiasis renal. Arch Esp Urol 1984;37:123-33.

29. Dauncey MJ, Widdowson EM. Urine excretion of calcium, magnesium, sodium and potassium in hard and soft water. Lancet 1972;1:711-4.

30. Breslau NA, McGuire JL, Zerweckh JE, Pak CYC. The role of dietary sodium on renal excretion and intestinal absorption of calcium and vitamin D metabolism. J Clin Endocrinol Metab 1982;55:369-73.
31. Moore ES, Coe FL, McMann BJ, Favus MJ. Idiopathic hypercalciuria in children: prevalence and metabolic characteristics. J Pediat 1978;92[Suppl 6]:906-10.

32. Zancheta JR, Bogado CE. Excreción urinaria de sodio y magnesio en la hipercalciuria idiopatica. Medicina, Buenos Aires 1991;51:296-302.

33. Akashi S, Motizuki H. Screening for hypercalciuria. Acta Paediatr Jpn 1990;32:701-9.

34. Berg C, Larson L, Tiselius HG. The composition of four-hour urine sample from patients with calcium oxalate stone disease. Br J Urol 1987;60:301-6.

35. Zollner N. Influence of various purines on uric acid metabolism. Nutr Diet 1973;19:34-7.

36. Sánchez-Bayle M, Ramo-Mancheño C. Hyperuricosuria and microhematuria in childhood. Pediatr Forum 1989;143:878-9.

\section{Endereço para correspondência:}

Dra. Maria Goretti M.G. Penido

Rua Tomé de Souza, 1292 - apto. 101 - Lourdes

CEP 30140-131, Belo Horizonte, MG

Fone: (31) 3225.2885 - Fone/fax: (31) 3241.4466

E-mail: gabrielp@gold.com.br 